\title{
BMJ Open Markers of gastrointestinal permeability and dysbiosis in premenopausal women with PCOS: a case-control study
}

\author{
Shilpa Lingaiah, ${ }^{1}$ Riikka K Arffman, ${ }^{1}$ Laure Morin-Papunen, ${ }^{1}$ \\ Juha S Tapanainen (D) , ${ }^{1,2}$ Terhi Piltonen ${ }^{1}$
}

To cite: Lingaiah S, Arffman RK, Morin-Papunen L, et al. Markers of gastrointestinal permeability and dysbiosis in premenopausal women with PCOS: a casecontrol study. BMJ Open 2021;11:e045324. doi:10.1136/ bmjopen-2020-045324

- Prepublication history and additional online supplemental material for this paper are available online. To view these files, please visit the journal online. To view these files, please visit the journal online (http://dx.doi.org/10.1136/ bmjopen-2020-045324).

JST and TP are joint senior authors.

Received 28 September 2020 Accepted 21 June 2021

Check for updates

(c) Author(s) (or their employer(s)) 2021. Re-use permitted under CC BY-NC. No commercial re-use. See rights and permissions. Published by BMJ.

${ }^{1}$ Department of Obstetrics and Gynaecology, PEDEGO Research Unit, Medical Research Centre, Oulu University Hospital, University of Oulu, Oulu, Finland ${ }^{2}$ Department of Obstetrics and Gynaecology, University of Helsinki and Helsinki University Central Hospital, Helsinki,

Finland

Correspondence to

Professor Juha S Tapanainen;

juha.tapanainen@helsinki.fi

\section{ABSTRACT}

Objectives Altered intestinal permeability and gut barrier dysfunction have been suggested to play a role in the pathogenetic mechanism of polycystic ovary syndrome (PCOS), the most common endocrine and metabolic condition in reproductive-aged women. However, data on intestinal permeability and dysbiosis of the gut microbiota in PCOS is still limited, with conflicting results. To this end, the concentrations of gastrointestinal permeability and gut dysbiosis markers were analysed in women with PCOS.

Design Case-control study.

Setting General community.

Participants 104 women with PCOS and 203 body mass index (BMI) matched control women at age 46.

Primary and secondary outcome measures Serum levels of zonulin, fatty acid-binding protein 2 (FABP2), urinary levels of indican, and hormonal and metabolic parameters.

Results Serum levels of zonulin $(128.0 \pm 17.0$ vs $130.9 \pm 14.0 \mathrm{ng} / \mathrm{mL}, \mathrm{p}=0.13)$ and FABP2 $(1.5 \pm 0.9$ vs $1.5 \pm 0.7 \mathrm{ng} / \mathrm{mL}, \mathrm{p}=0.63$ ) and urinary levels of indican $(9.5 \pm 5.5$ vs $8.4 \pm 4.2 \mathrm{mg} / \mathrm{dL}, \mathrm{p}=0.07)$ were comparable in women with PCOS and controls in the whole study population. Likewise, when the study population was divided into different BMl groups as normal weight, overweight and obese, the levels of the above markers were comparable between the study groups. After BMI adjustment, zonulin levels correlated with the levels of high-sensitivity $\mathrm{C}$ reactive protein and homoeostasis model assessment of insulin resistance $(p<0.05)$ both in women with PCOS and controls.

Conclusions Intestinal permeability markers zonulin and FABP2, and the dysbiosis marker indican do not seem to be altered in women with PCOS at age 46 compared with BMI-matched controls. Serum zonulin levels correlated with BMI, insulin resistance and inflammatory marker levels, but did not segregate women with PCOS and controls. This suggests that metabolic factors, but not PCOS per se, is the driving force of dysbiosis in premenopausal women with PCOS.

\section{INTRODUCTION}

The intestinal epithelium acts as a selectively permeable barrier, allowing absorption of nutrients and water while maintaining effective defence against micro-organisms and toxins. Intestinal mucosal permeability is

\section{Strengths and limitations of this study}

This is the first study to report on fatty acid-binding protein 2 and indican in women with polycystic ovary syndrome (PCOS), and the largest study by far to report on serum levels of zonulin in women with PCOS.

- This is the first study in which the study subjects have been matched for body mass index and age as regards the measurement of gastrointestinal permeability markers in women with PCOS.

- Urinary indican used for the assessment of dysbiosis is a surrogate marker and may not reflect the actual microbiome status.

- The age of the study subjects at the time of sampling was 46 years, and the metabolic profile did not differ significantly between women with PCOS and controls.

- The diagnosis of PCOS was based on self-reported symptoms of oligo-amenorrhoea and hirsutism. Ovarian ultrasonography data were not available.

mediated via junctional complexes, including tight-junction proteins. In recent years, disturbances of the intestinal barrier and increased intestinal permeability in various diseases have become evident. Indeed, increased intestinal permeability, often referred to as 'leaky gut', has been thought to be a common underlying mechanism in the pathogenesis of several inflammatory and autoimmune disorders. $^{12}$

Polycystic ovary syndrome (PCOS) is the most common endocrine disorder, affecting $6 \%-15 \%$ of reproductive-aged women. ${ }^{3}$ It is a complex disorder with heterogeneity of characteristics, including chronic anovulation, hyperandrogenism, obesity and insulin resistance, all having significant long-term metabolic and cardiovascular health implications. Even though metabolic, neuroendocrine and genetic factors have been suggested to play important roles in the development of PCOS, ${ }^{4}$ the underlying aetiology remains unclear. It has been hypothesised that gut 
barrier dysfunction may play a role in the pathogenesis of PCOS. Disturbances in intestinal bacterial flora cause increased intestinal mucosal permeability, with a consequent increase in lipopolysaccharide passage into the systemic circulation, which is thought to trigger a chronic inflammatory response and subsequent insulin resistance, and increased ovarian androgen production. ${ }^{5}$ Furthermore, chronic low-grade inflammation is often present in women with PCOS. ${ }^{6}$ In addition, ovarian theca cells, responsible for ovarian androgen synthesis, have been shown to have an altered steroid-hormone response in the presence of an inflammatory environment, linking low-grade inflammation to hyperandrogenism. ${ }^{7}$

The most commonly accepted method of assessing intestinal permeability is measurement of the urinary excretion ratio of lactulose/rhamnose or lactulose/ mannitol. ${ }^{8}$ Even though considered as an accurate, repeatable and non-invasive method, it does, however, have some limitations. It requires ingestion of probes and a period of fasting urine collection. Furthermore, analysis of urinary ratios is complex and time-consuming. Alternatively, assessment of intestinal permeability is possible by measuring the levels of biomarkers of intestinal barrier integrity. ${ }^{2}$

Zonulin, a human protein analogue of Vibrio choleraederived zonula occludens toxin, is the only physiological mediator known to reversibly regulate intestinal permeability by modulating intercellular tight junctions. ${ }^{9}$ Zonulin induces tight-junction disassembly, consequently increasing permeability of the intestinal epithelia and activating immune reactions. ${ }^{10}$ Zonulin is considered to be a non-invasive biomarker of intestinal permeability, and serum levels of zonulin have been shown to strongly correlate with the lactulose/mannitol ratio. ${ }^{10} 11$ Studies have shown that zonulin levels are increased in obesity, type 2 diabetes, gestational diabetes mellitus and positively correlated with insulin resistance and levels of inflammatory markers such as interleukin-6. ${ }^{12-14}$

Fatty acid-binding protein 2 (FABP2), also known as intestinal (I)-FABP, is an intracellular protein involved in the uptake and metabolism of fatty acids, and it is predominantly expressed in small intestinal enterocytes. FABP2 is released into the circulation following small intestinal mucosal injury, and elevated levels indicate intestinal epithelial cell damage. ${ }^{15}$ Since intestinal epithelial cell injury is considered a key pathway in altered intestinal permeability, ${ }^{16}$ FABP2 can be used as an indirect measure of increased intestinal permeability. Elevated FABP2 levels have been reported in coeliac disease and necrotising enterocolitis. ${ }^{17}{ }^{18}$ However, data are limited as regards metabolic diseases. Increased FABP2 levels have been reported in severely obese subjects with chronic hyperglycaemia. ${ }^{19}$

Changes in the composition of gut microflora have been associated with chronic diseases such as obesity, type 2 diabetes mellitus and metabolic syndrome. ${ }^{2021}$ Furthermore, recent studies have suggested that hyperandrogenaemia in PCOS may be related to changes in the gut microbiota or gut dysbiosis. ${ }^{22}$ The growth of gut microbiota is associated with the release of bacterial metabolites, which enter the systemic circulation via intestinal absorption and are excreted in the urine. ${ }^{23}$ One such metabolite is indican, also known as indoxyl sulfate. Dietary tryptophan is metabolised into indole by the gut microbiota, which after systemic absorption, is sulfated in the liver to indoxyl sulfate, and is excreted in the urine. Determining the urinary concentrations of indican can be used as a simple measure to assess dysbiosis.

Given that previous studies reporting gastrointestinal permeability markers in women with PCOS have performed the comparisons using controls not matched for age or body mass index (BMI), the aim of the present study was to determine the levels of these markers in women with PCOS and in BMI-matched and age-matched controls in a large community-based population. We further aimed to evaluate the possible associations of these gastrointestinal permeability markers with hormonal and metabolic parameters. In addition, concentrations of urinary indican, as a surrogate marker of altered gut microbiota, were also analysed.

\section{MATERIALS AND METHODS \\ Study population}

The study population consisted of women of the same age (46 years): 104women with PCOS and 203 BMI-matched non-PCOS controls from the prospective Northern Finland Birth Cohort 1966 (NFBC1966). Data collection in connection with NFBC1966 included postal questionnaires and clinical health examinations at 31 and 46 years. The questionnaire at age 31 included questions on oligo/amenorrhoea and hirsutism $(\mathrm{OA}+\mathrm{H})$ : 'Is your menstrual cycle often (more than twice a year) longer than 35 days?' and 'Do you have bothersome, excessive body-hair growth?' The questionnaire at age 46 included the question 'Have you ever been diagnosed as having polycystic ovaries and/or PCOS?' Accordingly, PCOS was diagnosed in women reporting $\mathrm{OA}+\mathrm{H}$ at age 31 and/or having a diagnosis of PCOS by age 46 . Women without $\mathrm{OA}+\mathrm{H}$ at age 31 and without diagnosis of PCOS by age 46 were classified as controls. The NFBC1966 flow chart is shown in figure 1 . The PCOS population has been validated and published previously. ${ }^{24-26}$ Only the women who had a full set of serum, urine and faecal samples available were included in the present study. The BMI-matched control women were included at a ratio of 1:2 (PCOS: controls). The subjects included in the present study had not been on any antibiotics within the previous 3 months at the time of sample collection.

The study participants provided written informed consent for the study and took part on a voluntary basis.

\section{Patient and public involvement statement}

The patients, the public or any third parties were not involved in the design, conduct, reporting or dissemination of our research. 


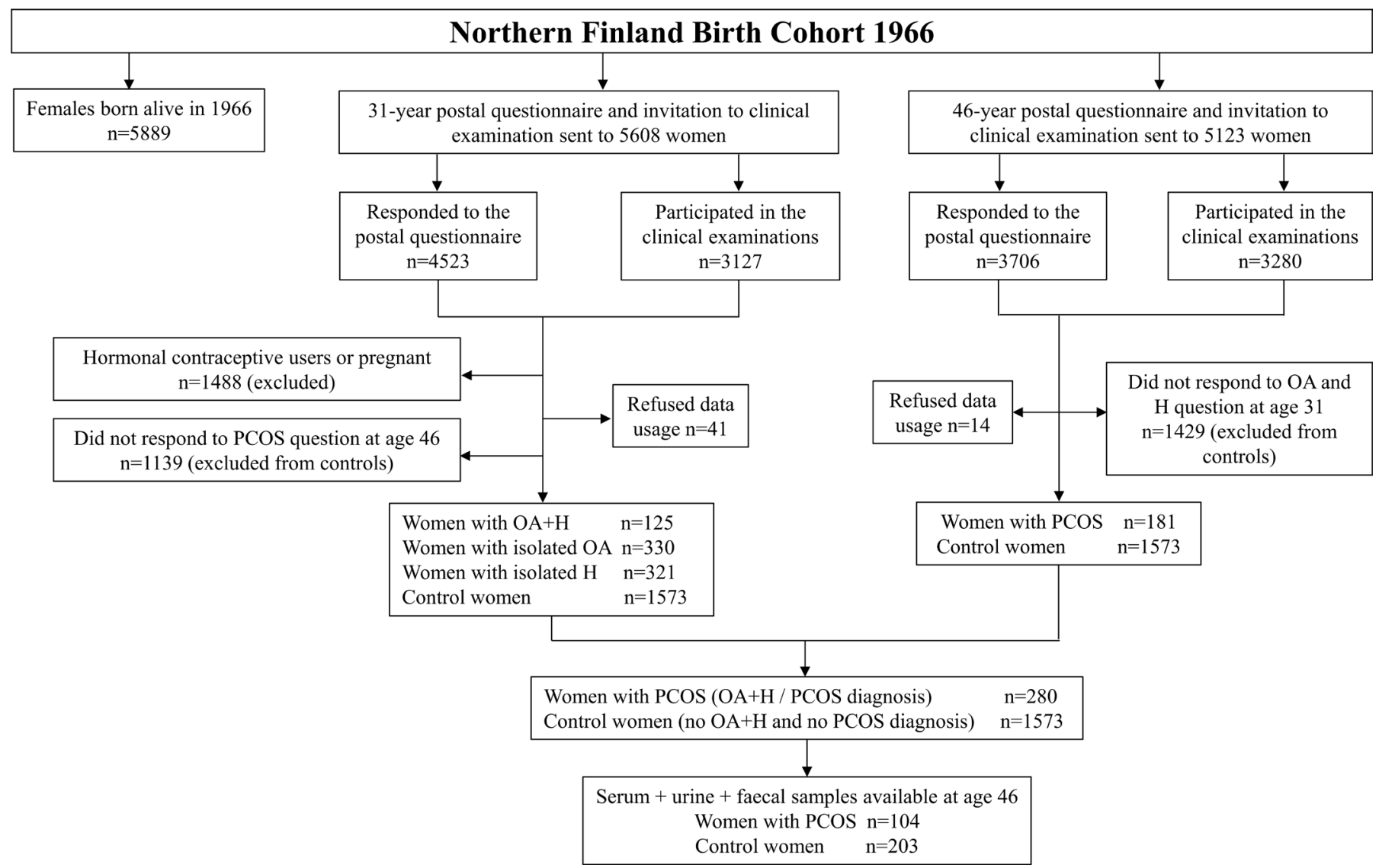

Figure 1 Flow chart of the study. $\mathrm{OA}+\mathrm{H}$, oligo/amenorrhoea and hirsutism; PCOS, polycystic ovary syndrome.

\section{Methods}

The study has been conducted and reported applying the criteria of the Strengthening the Reporting of Observational Studies in Epidemiology statement. ${ }^{27}$

\section{Anthropometric measurements}

All clinical assessments were performed by an experienced research nurse. Weight and height were measured at the time of clinical examination at age 46. BMI was calculated as the ratio of weight $(\mathrm{kg})$ and height squared $\left(\mathrm{m}^{2}\right)$. Waist and hip circumferences were measured (at the level midway between the lowest rib margin and the iliac crest, and at the widest part of the gluteal region respectively) and the waist-to-hip ratio (WHR) was calculated.

\section{Lifestyle and social characteristics}

Categorisation of lifestyle variables and social descriptives was based on the responses to the postal questionnaire. Smoking was categorised as non-smoker, former/ occasional smoker or active smoker. Alcohol consumption was categorised into three groups: abstainer, lowrisk drinker $(\leq 20 \mathrm{~g} /$ day $)$ or high-risk drinker $(>20 \mathrm{~g}$ / day). ${ }^{28}{ }^{29}$ Leisure time physical activity (PA) was selfreported with questions on the frequency and duration of light and brisk PAs, as described previously. ${ }^{30} \mathrm{PA}$ was calculated as metabolic equivalent of task (MET) scores in hours per week from the reported frequency and duration of leisure-time activities (light PA=3 METs, and brisk $\mathrm{PA}=5 \mathrm{METs}) .{ }^{31}$ Marital status was categorised as either single or in a relationship and education level was classified into three categories as basic, secondary or tertiary (online supplemental table 1 ).

\section{Oral glucose tolerance test}

A standard 2-hour oral glucose tolerance test (OGTT) was performed after an overnight fast (12 hours). Plasma glucose and serum insulin were measured at baseline and at 30, 60 and $120 \mathrm{~min}$ after a $75 \mathrm{~g}$ glucose intake. Glucose tolerance was classified according to American Diabetes Association guidelines as normal glucose tolerance (NGT) (fasting glucose $\leq 5.5 \mathrm{mmol} / \mathrm{L}$ and 2 hours glucose $<7.8 \mathrm{mmol} / \mathrm{L}$ ), impaired fasting glucose (IFG) (fasting glucose $5.6-6.9 \mathrm{mmol} / \mathrm{L}$ and normal 2-hour glucose) and impaired glucose tolerance (IGT) (normal fasting glucose and 2 hours glucose $7.8-11.0 \mathrm{mmol} / \mathrm{L}$ ).

\section{Laboratory methods}

Serum concentrations of FABP2 and zonulin were analysed by ELISA (R\&D Systems, Minneapolis, USA, and Immundiagnostik AG, Bensheim, Germany) according to the manufacturers' instructions, with intra-assay and interassay coefficients of variation of $4.1 \%$ and $11.1 \%$ for FABP2, and $6 \%$ and $13.6 \%$ for zonulin. Urinary concentrations of indican were measured by colorimetric assay according to the instructions of the manufacturer (SigmaAldrich, Missouri, USA), with a detection range between 0.2 and $20 \mathrm{mg} / \mathrm{dL}$. 
Table 1 Anthropometric, hormonal and metabolic parameters in the whole study population

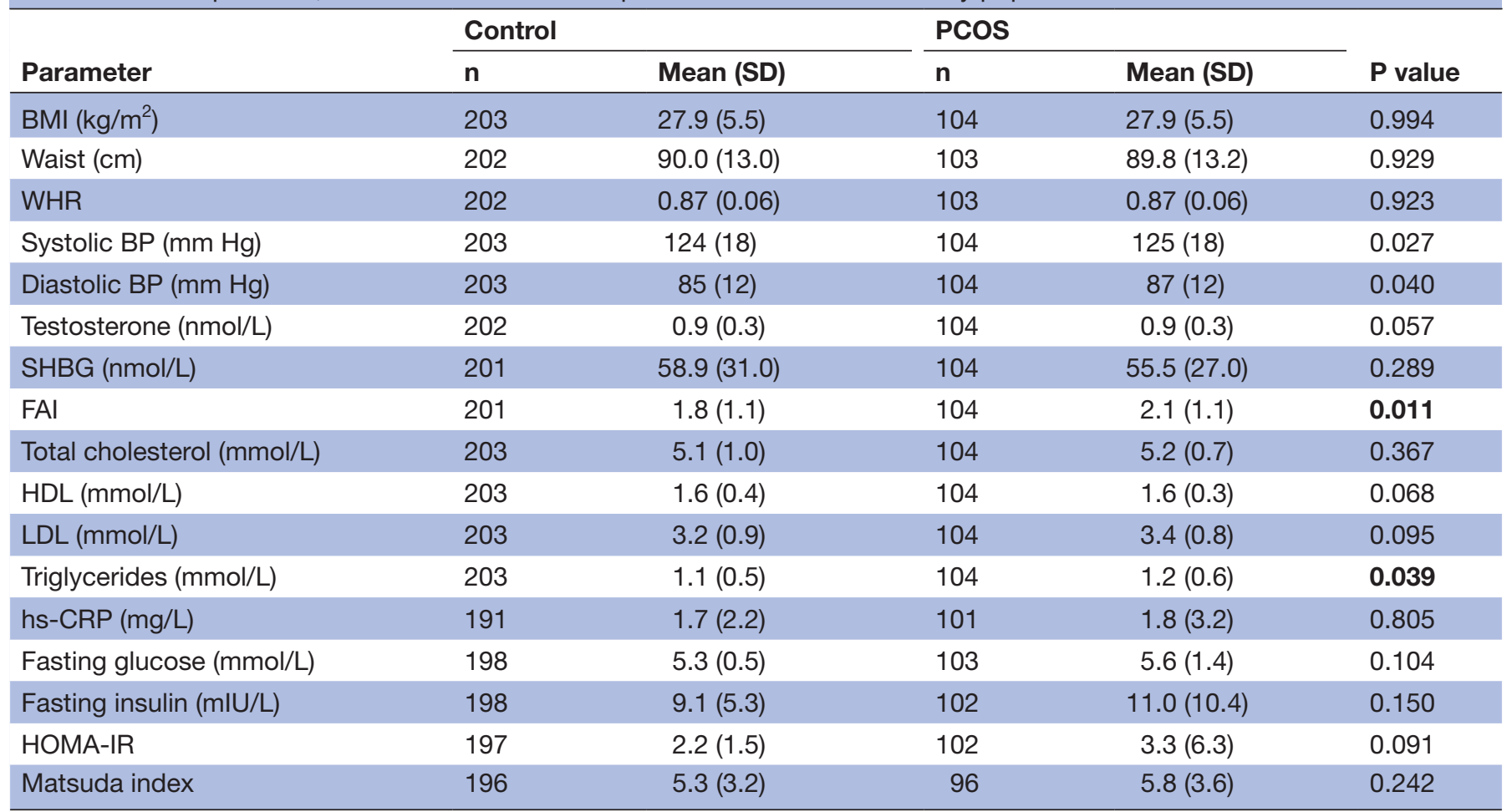

Data shown as mean (SD). Statistically significant $P$ values are in bold.

$P$ values according to independent samples t-tests.

BMI, body mass index; BP, blood pressure; FAl, Free Androgen Index; HDL, high-density lipoprotein; HOMA-IR, homoeostatic model assessment of insulin resistance; hs-CRP, high-sensitivity C reactive protein; LDL, low-density lipoprotein; SHBG, sex hormone-binding globulin; WHR, waist hip ratio.

Serum testosterone concentrations were analysed by using Agilent triple quadrupole 6410 liquid chromatography-mass spectrometry equipment with an electrospray ionisation source operating in positive-ion mode (Agilent Technologies, Wilmington, Delaware, USA). The normal upper limit for testosterone was $2.3 \mathrm{nmol} / \mathrm{L}$ at age 31 and $1.70 \mathrm{nmol} / \mathrm{L}$ at age 46 , based on 97.5 percentiles calculated in the women of the NFBC1966. Serum sex hormone-binding globulin (SHBG) concentrations were analysed by chemiluminometric immunoassay (Immulite 2000, Siemens Healthcare Diagnostics, Llanberis, UK). The Free Androgen Index was calculated by using the equation T/SHBG (both in $\mathrm{nmol} / \mathrm{L}$ ) $\times 100$.

Serum levels of total cholesterol, high-density lipoprotein (HDL), low-density lipoprotein (LDL) and triglycerides were determined by using an enzymatic assay method (Advia1800, Siemens Healthcare Diagnostics). High-sensitivity $\mathrm{C}$ reactive protein (hs-CRP) was analysed by nephelometric assay (BN ProSpec, Siemens Healthcare Diagnostics). Plasma glucose was analysed by an enzymatic dehydrogenase method (Advia 1800, Siemens Healthcare Diagnostics) and serum insulin by a chemiluminometric immunoassay (Advia Centaur XP, Siemens Healthcare Diagnostics). Insulin resistance was defined by the homoeostasis model assessment of insulin resistance (HOMA-IR) and insulin sensitivity by the composite Insulin Sensitivity Index or the Matsuda index, as described earlier. ${ }^{32}{ }^{33}$ All samples were analysed for hormonal and metabolic parameters at NordLab, Oulu (formerly known as Oulu University Hospital laboratory). Of note, the number of subjects varied between analyses owing to lack of measurements in some cases or non-response to some questions.

\section{Statistical analysis}

Statistical analyses were performed using SPSS V.25.0 software (IBM). Variables with a skewed distribution were logarithmically transformed before statistical analyses. Independent samples t-tests were used to assess differences between the PCOS and control groups. To evaluate the hormonal and metabolic changes in connection with BMI, the PCOS and control groups were subgrouped as normal weight $\left(\mathrm{BMI}<25 \mathrm{~kg} / \mathrm{m}^{2}\right)$, overweight $(25-30 \mathrm{~kg} /$ $\left.\mathrm{m}^{2}\right)$ and obese $\left(>30 \mathrm{~kg} / \mathrm{m}^{2}\right)$. One-way analysis of variance (ANOVA) and post hoc Tukey test was used to assess the possible BMI-related changes in the levels of gut permeability markers in the three different BMI subgroups. PA, calculated as MET score, was included in covariate analysis to control for its confounding effects on gastrointestinal permeability markers. Correlations between different parameters were assessed by using Pearson's correlation and adjustment for BMI was carried out by way of partial correlation analyses. The lowest $(<25$ th percentile) and highest ( $>75$ th percentile) quartiles for 
Table 2 Anthropometric, hormonal and metabolic parameters in the study population stratified according to different BMI categories

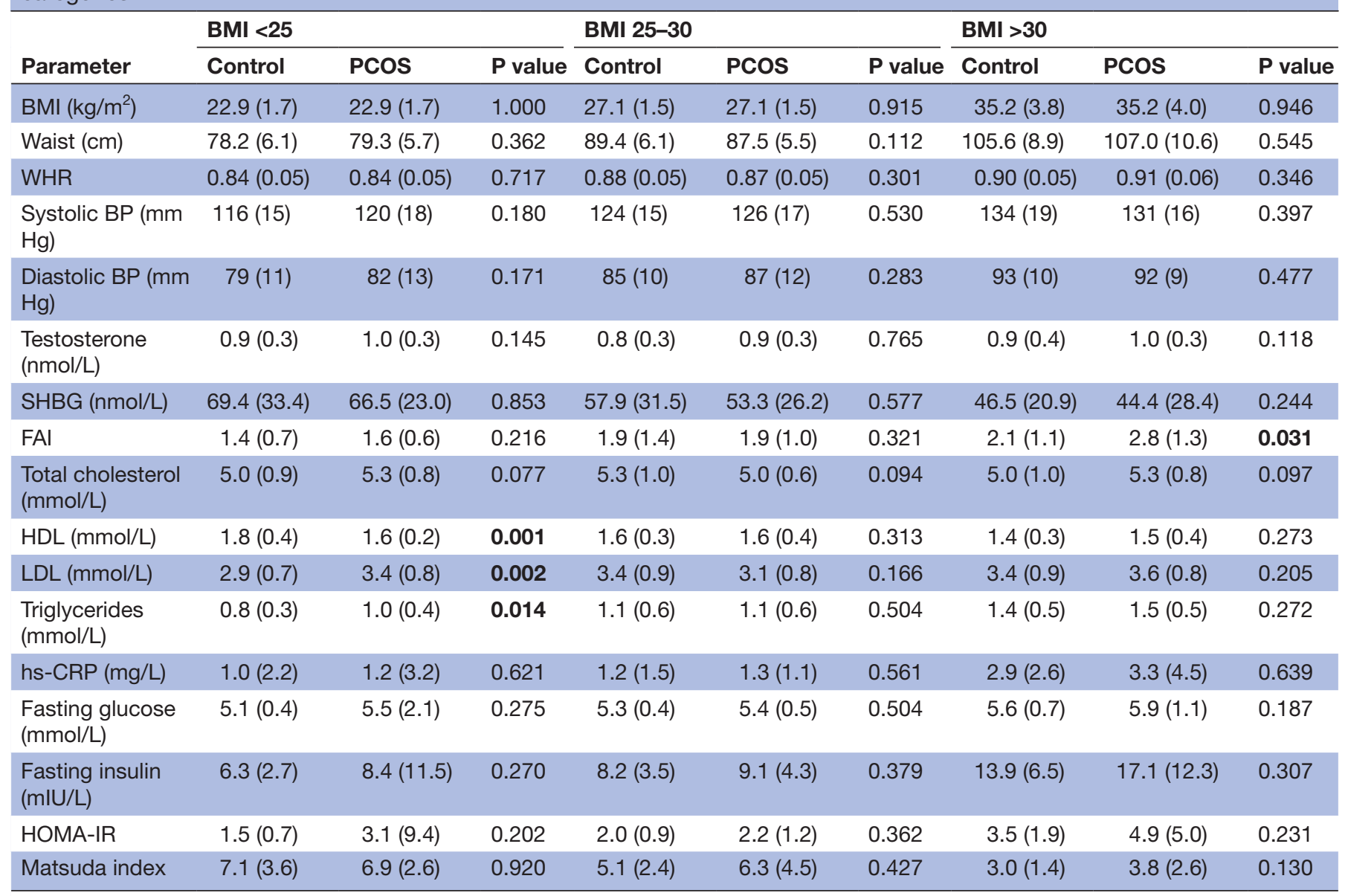

Data shown as mean (SD). Statistically significant $P$ values are in bold.

$P$ value according to independent samples t-test.

BMI, body mass index; BP, blood pressure; FAl, Free Androgen Index; HDL, high-density lipoprotein; HOMA-IR, homoeostatic model assessment of insulin resistance; hs-CRP, high-sensitivity $\mathrm{C}$ reactive protein; LDL, low-density lipoprotein; SHBG, sex hormone-binding globulin; WHR, waist hip ratio.

each gut permeability marker were estimated, and clinical and hormonal profiles of the study subjects were compared in each quartile. A value of $p<0.05$ was considered statistically significant.

\section{RESULTS}

\section{Characteristics of the study population}

The anthropometric, hormonal and metabolic parameters of the study population are shown in table 1. BMI matching was successful, as there was no difference in BMI between the respective study populations. Women with PCOS had higher levels of triglycerides and higher FAI values compared with the control women. The BMIstratified anthropometric, hormonal and metabolic parameters are shown in table 2. Normal-weight women with PCOS had decreased levels of HDL, and increased levels of LDL and triglycerides compared with normalweight controls. Obese women with PCOS had higher FAI values compared with obese controls.

\section{Concentrations of serum FABP2 and zonulin, and urinary} indican

Serum levels of FABP2 and zonulin and urinary levels of indican were comparable among women with PCOS and controls in the whole study population as well as in the different BMI groups (figure 2). BMI-stratified analysis using one-way ANOVA showed that serum concentrations of zonulin increased with BMI in both women with PCOS $(p<0.001)$ and controls $(p=0.001)$, while no changes were observed in serum levels of FABP2 or urinary levels of indican.

\section{Correlation analyses}

Serum levels of zonulin correlated positively with BMI, waist circumference and WHR in both control women and in women with PCOS, but remained statistically significant after adjusting for BMI only in control women (table 3). Moreover, in control women the levels of zonulin correlated with the levels of total cholesterol, LDL, triglycerides, FABP2 and indican, and the Matsuda 

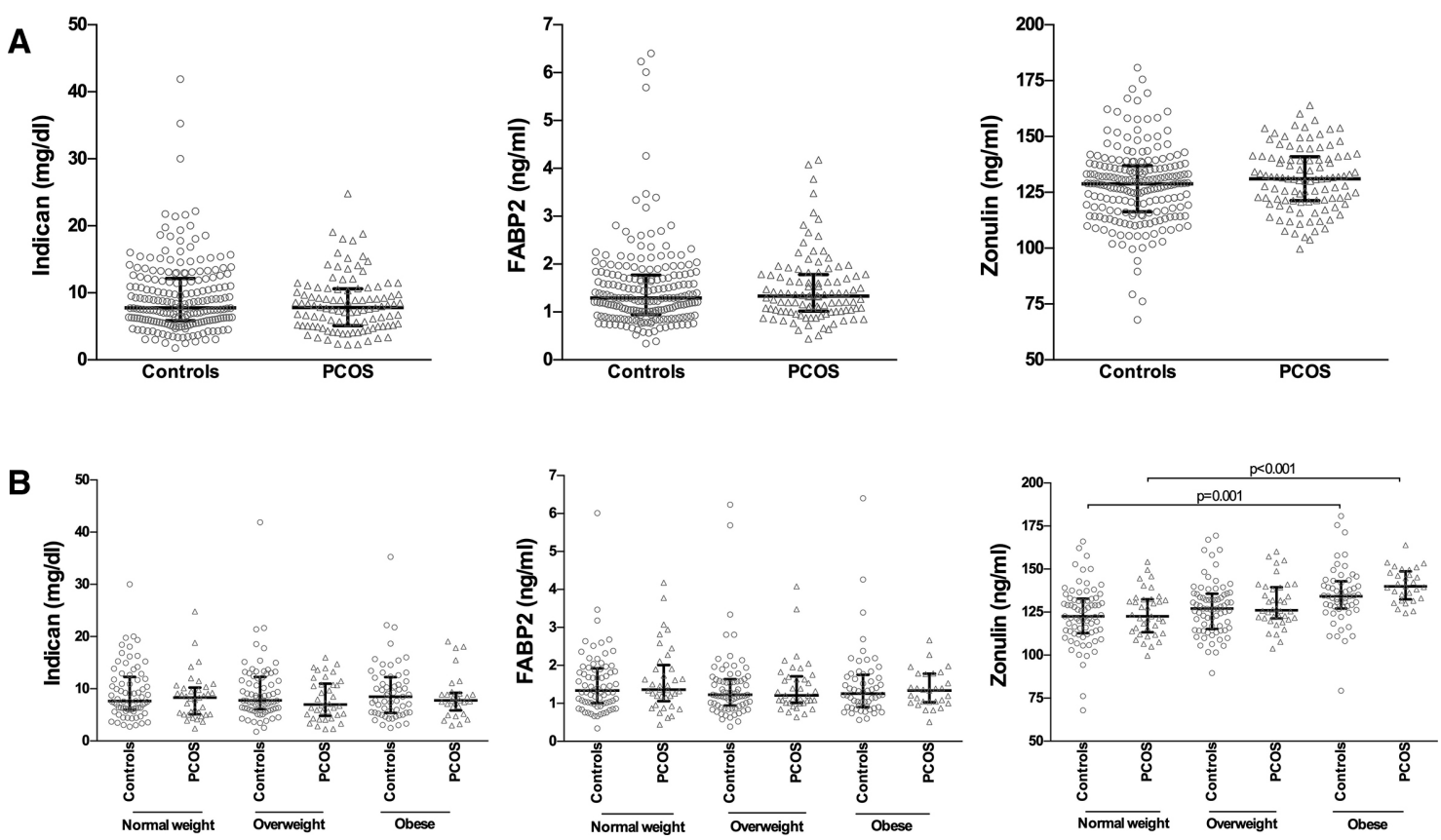

Figure 2 Concentrations of indican, FABP2 and zonulin in (A) whole study population (B) women stratified according to $\mathrm{BMl}$ as normal weight, overweight and obese $\left(\mathrm{BMl}<25,25-30\right.$ and $>30 \mathrm{~kg} / \mathrm{m}^{2}$, respectively). The horizontal line in each plot corresponds to the median value. The vertical line represents the 25th-75th percentile range. $P$ values according to one-way ANOVA in controls and women with PCOS. ANOVA, analysis of variance; BMI, body mass index; FABP2, fatty acid-binding protein 2; PCOS, polycystic ovary syndrome.

index after adjustment for BMI. Furthermore, in both control women and women with PCOS, serum levels of zonulin correlated positively with levels of hs-CRP, fasting glucose, fasting insulin and HOMA-IR after adjustment for BMI.

Concentrations of FABP2 were negatively correlated with those of testosterone $(\mathrm{r}=-0.220, \mathrm{p}=0.002)$, and with the FAI $(r=-0.228, p=0.001)$ in the control women after adjusting for BMI. No significant correlations between the levels of FABP2, indican and other parameters were observed in women with PCOS (data not shown).

\section{Concentrations of urinary indican, serum FABP2 and zonulin based on androgen status, glucose tolerance and metabolic syndrome}

When women with PCOS were classified on the basis of their androgenic status as normoandrogenic or hyperandrogenic, the levels of indican $(8.4 \pm 3.4($ mean \pm SD) $)$ vs $8.4 \pm 5.0 \mathrm{mg} / \mathrm{dL})$, FABP2 $(1.5 \pm 0.7$ vs $1.5 \pm 0.7 \mathrm{ng} / \mathrm{mL})$ and zonulin $(129.5 \pm 12.4$ vs $132.7 \pm 5.7 \mathrm{ng} / \mathrm{mL})$ were comparable in the two groups. No significant differences were found in the levels of indican, FABP2 or zonulin when the women were classified on the basis of their glucose tolerance as NGT, IGT or IFG (data not shown). Furthermore, when women with PCOS were classified on the basis of their metabolic syndrome status ${ }^{34}$ no significant differences were found in the levels of indican, FABP2 or zonulin (data not shown).

\section{Quartile analysis}

When quartiles of hormonal and metabolic parameters in women with PCOS were compared, testosterone levels were increased in the lower indican quartile compared with the highest quartile $(1.0 \pm 0.3$ vs $0.8 \pm 0.4 \mathrm{nmol} / \mathrm{L}$; $\mathrm{p}=0.030$ ), whereas no differences were observed as regards FABP2. Significant differences were observed in anthropometric, hormonal and metabolic parameters as regards zonulin quartiles (online supplemental table 2).

\section{DISCUSSION}

To the best of our knowledge, this is the first study to report on FABP2 and indican in women with PCOS, and the largest study to report on serum levels of zonulin in women with PCOS. Moreover, this is also the first study in which the study subjects have been matched for BMI and age. The present study showed that serum levels of zonulin and FABP2, and urinary levels of indican were comparable in women with PCOS and non-PCOS control women at age 46. Furthermore, serum concentrations of zonulin increased with BMI in both women with PCOS and in controls, while no changes were observed in serum levels of FABP2 or urinary levels of indican.

Data on intestinal permeability in women with PCOS and its role in the pathogenesis of the condition is limited. Previous, although few, studies have analysed gut permeability in women with PCOS by determining serum levels of zonulin and reported conflicting results. In line with the present results, in one study, no differences in the levels of serum zonulin were reported in women with PCOS without metabolic syndrome, compared with controls. ${ }^{35}$ In contrast, Zhang et al reported elevated levels of serum zonulin in women with PCOS compared with healthy 
Table 3 Correlations between zonulin and various parameters in the study population

\begin{tabular}{|c|c|c|c|c|}
\hline \multirow[b]{2}{*}{ Parameter } & \multicolumn{2}{|c|}{ Controls } & \multicolumn{2}{|l|}{ PCOS } \\
\hline & $\mathbf{R}$ & $P$ value & $\mathbf{R}$ & $P$ value \\
\hline BMI & 0.268 & $<0.001$ & 0.512 & $<0.001$ \\
\hline Waist & 0.314 & $<0.001^{*}$ & 0.507 & $<0.001$ \\
\hline WHR & 0.286 & $<0.001^{*}$ & 0.320 & 0.001 \\
\hline Testosterone & 0.031 & 0.658 & 0.159 & 0.106 \\
\hline SHBG & -0.154 & 0.029 & -0.191 & 0.053 \\
\hline FAI & 0.126 & 0.074 & 0.277 & 0.004 \\
\hline Total cholesterol & 0.148 & $0.035^{\star}$ & 0.171 & 0.083 \\
\hline HDL & -0.145 & 0.039 & -0.104 & 0.291 \\
\hline LDL & 0.229 & $0.001^{*}$ & 0.213 & 0.030 \\
\hline Triglycerides & 0.360 & $<0.001^{*}$ & 0.385 & $<0.001$ \\
\hline hs-CRP & 0.269 & $<0.001^{*}$ & 0.409 & $<0.001^{*}$ \\
\hline Fasting glucose & 0.257 & $<0.001^{\star}$ & 0.292 & $0.003^{\star}$ \\
\hline Fasting insulin & 0.377 & $<0.001^{*}$ & 0.459 & $<0.001^{*}$ \\
\hline HOMA-IR & 0.382 & $<0.001^{*}$ & 0.463 & $<0.001^{*}$ \\
\hline Matsuda index & -0.326 & $<0.001^{\star}$ & -0.320 & 0.002 \\
\hline FABP2 & 0.155 & $0.027^{\star}$ & 0.054 & 0.588 \\
\hline Indican & 0.159 & $0.024^{*}$ & -0.029 & 0.769 \\
\hline
\end{tabular}

${ }^{*} \mathrm{P}<0.05$ after adjustment for BMI.

BMI, body mass index; FABP2, fatty acid-binding protein 2; FAI, Free Androgen Index; HDL, high-density lipoprotein; HOMA-IR, homoeostatic model assessment of insulin resistance; hs-CRP, high sensitivity $C$ reactive protein; LDL, low-density lipoprotein; PCOS, polycystic ovary syndrome; SHBG, sex hormone-binding globulin; WHR, waist-hip ratio.

controls. ${ }^{36}$ Of note, there were significant differences in the metabolic profiles of women with PCOS compared with the controls in their study, whereas in the present study metabolic profiles were comparable in the study groups. This may reflect the greater age of subjects in the present study and the possibility that the groups may become metabolically closer to each other with advancing age.

It has been reported that obesity is associated with increased intestinal permeability ${ }^{37} 38$ and studies have shown elevated levels of serum zonulin in obese subjects when compared with non-obese. ${ }^{12}{ }^{39}$ In line with these results, our study showed increased levels of serum zonulin with increasing BMI in both women with PCOS and controls. The highest levels were observed in obese women when compared with normal-weight women, suggesting that serum levels of zonulin may be determined by the degree of obesity, not PCOS per se.

Insulin resistance is a common feature in women with PCOS. ${ }^{40}$ Few studies have examined the association of serum zonulin levels with insulin resistance, but the results have been conflicting; both no correlation ${ }^{35}$ and a positive correlation ${ }^{12}{ }^{36}$ have been reported. In the present study, serum zonulin levels were correlated with HOMA-IR values in both women with PCOS and in controls after adjustment for BMI. Furthermore, the levels of hs-CRP correlated with those of zonulin in both study groups, suggesting that zonulin may be a marker of insulin resistance and inflammation.

There have been no previous studies in which the levels of FABP2, another marker of intestinal permeability, have been assessed in women with PCOS. Serum levels of FABP2 were similar in women with PCOS and controls, and there was no correlation between concentrations of FABP2 and hs-CRP. It has been suggested that FABP2 is not a direct marker of altered intestinal permeability per se, but a marker of enterocyte injury, and enterocyte damage contributes to altered intestinal barrier function. ${ }^{16}$ Our findings suggest that there was no altered intestinal permeability in our PCOS study population, as reflected in the levels of gastrointestinal permeability markers. This may indicate not only that disturbances in intestinal permeability are not associated with the pathogenesis of PCOS, but also the fact that the women in our study did not present with extreme metabolic and/or hormonal dysfunction.

Levels of urinary indican, a marker of dysbiosis, were comparable in women with PCOS and controls, suggesting that dysbiosis of the gut microbiota might not be linked to PCOS in our study population. This is supported by the findings of a microbiome study conducted in the same cohort, which revealed no differences in the gut microbiome profile between women with PCOS and control women at late reproductive age. ${ }^{41}$ This is in contrast to the results of studies indicating an association between gut microbiota and PCOS. ${ }^{22} 42$ However, it has to be noted that urinary indican is only a surrogate marker in the assessment of dysbiosis and may not reflect the actual status of the microbiome.

There are strengths as well as limitations in the present study. The study population was homogeneous, with all participants being Caucasian and from the unique NFBC 1966. Our study included BMI-matched and age-matched women with PCOS and controls, thus minimising the effects of BMI and age on case-control comparisons. The fact that the diagnosis of PCOS was based on questionnaire data and no ovarian ultrasonography was performed can be considered as a limitation. Women with relatively mild PCOS symptoms could have been included in the study population, possibly decreasing differences between the study groups. However, it has been shown previously that self-reported $\mathrm{OA}+\mathrm{H}$ can identify women with the typical endocrine and metabolic profiles of PCOS. ${ }^{24-26}$ The data may also have a selection bias as only those women with PCOS who had faecal samples were selected from the NFBC1966 for the present study. The controls, however, were chosen to match the BMI of women with PCOS, which minimised the confounding effects of BMI. Due to the matching, the control women also had relatively high BMIs, and consequently more metabolic disturbances, which may narrow differences between the groups. The age of the study subjects at the time of sampling was 46 
years and thus some women may have been premenopausal. However, given that menopausal age is delayed in PCOS, the effect of menopause, at least among women with PCOS, was most likely minimal. ${ }^{4344}$

In conclusion, serum levels of gastrointestinal permeability markers, namely zonulin and FABP2, and levels of urinary indican, a marker of dysbiosis, did not differ between BMI-matched women with PCOS and controls at late reproductive age. Zonulin presented as a metabolic and inflammatory marker, although its concentrations did not associate with PCOS.

Acknowledgements We thank late Professor Paula Rantakallio for establishing the NFBC, the participants in the 46-year study, and the NFBC Project Centre. We also thank Nick Bolton for revising the language.

Contributors SL: study design, data analyses and interpretation, manuscript writing; RKA: study design, sample coordination, data interpretation; LMP: data interpretation; JST: study design, data interpretation; TP: study design, data interpretation. All authors revised the manuscript.

Funding This study was funded by grants from the Academy of Finland, the Sigrid Jusélius Foundation, Oulu University Scholarship Foundation, Helsinki University Hospital and Oulu University Hospital Research Funds, Medical Research Centre Oulu, Oulu University Hospital and University of Oulu.

Competing interests None declared.

Patient consent for publication Not required.

Ethics approval The study was approved by the Ethics Committee of the Northern Ostrobothnia Hospital District in Oulu, Finland and conducted according to the Declaration of Helsinki.

Provenance and peer review Not commissioned; externally peer reviewed. Data availability statement Data are available on reasonable request.

Supplemental material This content has been supplied by the author(s). It has not been vetted by BMJ Publishing Group Limited (BMJ) and may not have been peer-reviewed. Any opinions or recommendations discussed are solely those of the author(s) and are not endorsed by BMJ. BMJ disclaims all liability and responsibility arising from any reliance placed on the content. Where the content includes any translated material, BMJ does not warrant the accuracy and reliability of the translations (including but not limited to local regulations, clinical guidelines, terminology, drug names and drug dosages), and is not responsible for any error and/or omissions arising from translation and adaptation or otherwise.

Open access This is an open access article distributed in accordance with the Creative Commons Attribution Non Commercial (CC BY-NC 4.0) license, which permits others to distribute, remix, adapt, build upon this work non-commercially, and license their derivative works on different terms, provided the original work is properly cited, appropriate credit is given, any changes made indicated, and the use is non-commercial. See: http://creativecommons.org/licenses/by-nc/4.0/.

ORCID iD

Juha S Tapanainen http://orcid.org/0000-0002-3139-9128

\section{REFERENCES}

1 Groschwitz KR, Hogan SP. Intestinal barrier function: molecular regulation and disease pathogenesis. J Allergy Clin Immunol 2009;124:3-2.

2 Bischoff SC, Barbara G, Buurman W, et al. Intestinal permeability--a new target for disease prevention and therapy. BMC Gastroenterol 2014;14:189.

3 March WA, Moore VM, Willson KJ, et al. The prevalence of polycystic ovary syndrome in a community sample assessed under contrasting diagnostic criteria. Hum Reprod 2010;25:544-51.

4 Dumesic DA, Oberfield SE, Stener-Victorin E, et al. Scientific statement on the diagnostic criteria, epidemiology, pathophysiology, and molecular genetics of polycystic ovary syndrome. Endocr Rev 2015;36:487-525.

5 Tremellen K, Pearce K. Dysbiosis of Gut Microbiota (DOGMA)--a novel theory for the development of Polycystic Ovarian Syndrome. Med Hypotheses 2012;79:104-12.
6 González F. Inflammation in polycystic ovary syndrome: underpinning of insulin resistance and ovarian dysfunction. Steroids 2012;77:300-5.

7 Fox CW, Zhang L, Sohni A, et al. Inflammatory stimuli trigger increased androgen production and shifts in gene expression in Theca-Interstitial cells. Endocrinology 2019;160:2946-58.

8 de Kort S, Keszthelyi D, Masclee AAM. Leaky gut and diabetes mellitus: what is the link? Obes Rev 2011;12:449-58.

9 Fasano A, Not T, Wang W, et al. Zonulin, a newly discovered modulator of intestinal permeability, and its expression in coeliac disease. Lancet 2000;355:1518-9.

10 Wang W, Uzzau S, Goldblum SE, et al. Human zonulin, a potential modulator of intestinal tight junctions. J Cell Sci 2000;113 Pt 24:4435-40.

11 Sapone A, de Magistris L, Pietzak M, et al. Zonulin upregulation is associated with increased gut permeability in subjects with type 1 diabetes and their relatives. Diabetes 2006;55:1443-9.

12 Moreno-Navarrete JM, Sabater M, Ortega F, et al. Circulating zonulin, a marker of intestinal permeability, is increased in association with obesity-associated insulin resistance. PLoS One 2012;7:e37160.

13 Demir E, Ozkan H, Seckin KD, et al. Plasma zonulin levels as a non-invasive biomarker of intestinal permeability in women with gestational diabetes mellitus. Biomolecules 2019;9:24.

14 Jayashree B, Bibin YS, Prabhu D, et al. Increased circulatory levels of lipopolysaccharide (LPS) and zonulin signify novel biomarkers of proinflammation in patients with type 2 diabetes. Mol Cell Biochem 2014;388:203-10.

15 Pelsers MMAL, Namiot Z, Kisielewski W, et al. Intestinal-Type and liver-type fatty acid-binding protein in the intestine. tissue distribution and clinical utility. Clin Biochem 2003;36:529-35.

16 March DS, Marchbank T, Playford RJ, et al. Intestinal fatty acidbinding protein and gut permeability responses to exercise. Eur $J$ Appl Physiol 2017;117:931-41.

17 Thuijls G, Derikx JPM, van Wijck K, et al. Non-invasive markers for early diagnosis and determination of the severity of necrotizing enterocolitis. Ann Surg 2010;251:1174-80.

18 Adriaanse MPM, Tack GJ, Passos VL, et al. Serum I-FABP as marker for enterocyte damage in coeliac disease and its relation to villous atrophy and circulating autoantibodies. Aliment Pharmacol Ther 2013;37:482-90.

19 Verdam FJ, Greve JWM, Roosta S, et al. Small intestinal alterations in severely obese hyperglycemic subjects. J Clin Endocrinol Metab 2011;96:379.

20 Tilg H, Kaser A. Gut microbiome, obesity, and metabolic dysfunction. $J$ Clin Invest 2011;121:2126-32.

21 Tilg $\mathrm{H}$, Moschen AR. Microbiota and diabetes: an evolving relationship. Gut 2014;63:1513-21.

22 Thackray VG, Sex TVG. Sex, microbes, and polycystic ovary syndrome. Trends Endocrinol Metab 2019;30:54-65.

23 Lord RS, Bralley JA. Clinical applications of urinary organic acids. Part 2. dysbiosis markers. Altern Med Rev 2008;13:292-306.

24 Taponen S, Martikainen H, Järvelin M-R, et al. Hormonal profile of women with self-reported symptoms of oligomenorrhea and/or hirsutism: Northern Finland birth cohort 1966 study. J Clin Endocrinol Metab 2003;88:141-7.

25 Taponen S, Ahonkallio S, Martikainen $\mathrm{H}$, et al. Prevalence of polycystic ovaries in women with self-reported symptoms of oligomenorrhoea and/or hirsutism: Northern Finland birth cohort 1966 study. Hum Reprod 2004;19:1083-8.

26 Taponen S, Martikainen $\mathrm{H}$, Järvelin M-R, et al. Metabolic cardiovascular disease risk factors in women with self-reported symptoms of oligomenorrhea and/or hirsutism: Northern Finland birth cohort 1966 study. J Clin Endocrinol Metab 2004;89:2114-8.

27 von Elm E, Altman DG, Egger M, et al. The strengthening the reporting of observational studies in epidemiology (STROBE) statement: guidelines for reporting observational studies. Int J Surg 2014;12:1495-9.

28 Laitinen J, Pietiläinen K, Wadsworth M, et al. Predictors of abdomina obesity among 31-y-old men and women born in northern Finland in 1966. Eur J Clin Nutr 2004:58:180-90.

29 Fawehinmi TO, llomäki J, Voutilainen S, et al. Alcohol consumption and dietary patterns: the FinDrink study. PLoS One 2012;7:e38607.

30 Kiviniemi AM, Perkiömäki N, Auvinen J, et al. Lifelong physical activity and cardiovascular autonomic function in midlife. Med Sci Sports Exerc 2016:48:1506-13.

31 Suija K, Timonen M, Suviola M, et al. The association between physical fitness and depressive symptoms among young adults: results of the Northern Finland 1966 birth cohort study. BMC Public Health 2013;13:535.

32 Matthews DR, Hosker JP, Rudenski AS, et al. Homeostasis model assessment: insulin resistance and beta-cell function from fasting 
plasma glucose and insulin concentrations in man. Diabetologia 1985;28:412-9.

33 Matsuda M, DeFronzo RA. Insulin sensitivity indices obtained from oral glucose tolerance testing: comparison with the euglycemic insulin clamp. Diabetes Care 1999;22:1462-70.

34 Rotterdam ESHRE/ASRM-Sponsored PCOS Consensus Workshop Group. Revised 2003 consensus on diagnostic criteria and longterm health risks related to polycystic ovary syndrome. Fertil Steril 2004;81:19-25

35 Cetin Z, Kosem A, Can B, et al. Serum zonulin level is not elevated in patients with polycystic ovary syndrome without metabolic syndrome. Arch Gynecol Obstet 2019;300:1785-90.

36 Zhang D, Zhang L, Yue F, et al. Serum zonulin is elevated in women with polycystic ovary syndrome and correlates with insulin resistance and severity of anovulation. Eur $\mathrm{J}$ Endocrinol 2015;172:29-36.

37 Lam YY, Ha CWY, Campbell CR, et al. Increased gut permeability and microbiota change associate with mesenteric fat inflammation and metabolic dysfunction in diet-induced obese mice. PLoS One 2012;7:e34233.
38 Gummesson A, Carlsson LMS, Storlien LH, et al. Intestinal permeability is associated with visceral adiposity in healthy women. Obesity 2011;19:2280-2.

39 Zak-Gołab A, Kocełak P, Aptekorz M, et al. Gut microbiota, microinflammation, metabolic profile, and zonulin concentration in obese and normal weight subjects. Int $J$ Endocrinol 2013;2013:674106.

40 Ovalle F, Azziz R. Insulin resistance, polycystic ovary syndrome, and type 2 diabetes mellitus. Fertil Steril 2002;77:1095-105.

41 Lüll K, Arffman RK, Sola-Leyva A, et al. The gut microbiome in polycystic ovary syndrome and its association with metabolic traits. $J$ Clin Endocrinol Metab 2021:106:858-71.

42 Liu R, Zhang C, Shi Y, et al. Dysbiosis of gut microbiota associated with clinical parameters in polycystic ovary syndrome. Front Microbiol 2017;8:324.

43 Forslund M, Landin-Wilhelmsen K, Schmidt J, et al. Higher menopausal age but no differences in parity in women with polycystic ovary syndrome compared with controls. Acta Obstet Gynecol Scand 2019;98:320-6.

$44 \mathrm{Li}$ J, Eriksson M, Czene K, et al. Common diseases as determinants of menopausal age. Hum Reprod 2016;31:2856-64. 